K. Nomizu and L. Rodríguez

Nagoya Math. J.

Vol. 48 (1972), 197-201

\title{
UMBILICAL SUBMANIFOLDS AND MORSE FUNCTIONS
}

\author{
KATSUMI NOMIZU AND LUCIO RODRÍGUEZ
}

Let $M^{n}$ be a differentiable manifold (of class $C^{\infty}$ ). By a Morse function on $M^{n}$ we mean a differentiable function whose critical points are all non-degenerate. If $f$ is an immersion of $M^{n}$ into a Euclidean space $R^{m}$, we may obtain Morse functions on $M^{n}$ in the following way. Let $p$ be a point of $R^{m}$ and define a differentiable function $L_{p}$ on $M^{n}$ by

$$
L_{p}(x)=d(p, f(x))^{2}, \quad x \in M^{n}
$$

where $d$ denotes the Euclidean distance in $R^{m}$. Then, for almost all $p \in R^{m}, L_{p}$ is a Morse function on $M^{n}$ (see [2], p. 36).

It is a well-known theorem of Reeb that if a compact differentiable manifold $M^{n}$ admits a Morse function with exactly two critical points, then $M^{n}$ is a topological sphere (see [2], p. 25). In the present note we shall prove the following results of a geometric nature (in contrast to a topological nature).

THEOREM A. Let $M^{n}$ be a connected compact differentiable manifold $(n \geqq 2)$ immersed in a Euclidean space $R^{m}$. If every Morse function on $M^{n}$ of the form $L_{p}, p \in R^{m}$, has exactly two critical points, then $M^{n}$ is imbedded as a Euclidean n-sphere.

Of course, a Euclidean $n$-sphere in $R^{m}$ means a hypersphere in a Euclidean $(n+1)$-subspace $R^{n+1}$ of $R^{m}$. As a matter of fact, Theorem A follows from the following more general result.

THEOREM B. Let $M^{n}, n \geqq 2$, be a connected, complete Riemannian manifold isometrically immersed in a Euclidean space $R^{m}$. If every Morse function on $M^{n}$ of the form $L_{p}, p \in R^{m}$, has index 0 or $n$ at any of its critical points, then $M^{n}$ is imbedded as a Euclidean $n$-subspace or a Euclidean $n$-sphere in $R^{m}$.

Received June 6, 1972.

Work supported by NSF Grant GP-28419. 
As another corollary, we obtain

THEOREM C. Under the assumptions of Theorem B, if the index is always 0, then $M^{n}$ is imbedded as a Euclidean $n$-subspace of $R^{m}$.

\section{Preliminaries.}

It is necessary to recall certain concepts and results on focal points, which can be found in [2, pp. 32-38]. Although this reference treats submanifolds imbedded in a Euclidean space, the same results hold for immersed submanifolds.

Let $f$ be an immersion of a differentiable manifold $M^{n}$ into a Euclidean space $R^{m}$. A point of the normal bundle $N$ of $M^{n}$ is denoted by $(x, \xi)$, where $x$ is a point of $M^{n}$ and $\xi$ is a vector normal to $f\left(M^{n}\right)$ at $f(x)$. Let $F$ be a differentiable mapping of $N$ into $R^{m}$ given by $F(x, \xi)=f(x)+\xi$. A point $p \in R^{m}$ is called a focal point of $M$ if $p=F(x, \xi)$, where $(x, \xi)$ is a point of $N$ where the Jacobian $F_{*}$ of $F$ is degenerate. In this case, we also say that $p$ is a focal point of $(M, x)$. By virtue of Sard's theorem, the set of focal points of $M$ has measure 0 .

It is known that a point $p=F(x, \xi)$, where $(x, \xi) \in N$, is a focal point of $(M, x)$ if and only if the endomorphism $I-A_{\xi}$ on the tangent space $T_{x}\left(M^{n}\right)$ is degenerate. Here $I$ is the identity transformation of $T_{x}\left(M^{n}\right)$ and $A_{\xi}$ is the symmetric endomorphism corresponding to the second fundamental form of $M$ at $x$ in the direction of $\xi$.

On the other hand, let $p \in R^{m}$ and consider the function $L_{p}(x)=$ $d(f(x), p)^{2}$ on $M^{n}$. A point $x \in M^{n}$ is a critical point of $L_{p}$ if and only if the vector $\xi$ from $f(x)$ to $p$ is normal to $f\left(M^{n}\right)$. In this case, the Hessian $H$ of $L_{p}$ at $x$, which is a bilinear symmetric function on $T_{x}(M)$ $\times T_{x}(M)$, is given by

$$
H(X, Y)=2\left\langle I-A_{\xi}(X), Y\right\rangle, \quad X, Y \in T_{x}\left(M^{n}\right),
$$

where $\langle$,$\rangle is the inner product on T_{x}(M)$ induced from the Euclidean metric in $R^{m}$ through the immersion $f$. Thus $H$ is degenerate at $x$ (i.e., $x$ is a degenerate critical point of $L_{p}$ ) if and only if $I-A_{\xi}$ is degenerate (i.e., $p$ is a focal point of $(M, x)$ ). If $x$ is a nondegenerate critical point of $L_{p}$, the index at $x$ is equal to the number of negative eigenvalues of $I-A_{\xi}$, counting multiplicities, in other words, the number of eigenvalues of $A_{\xi}$ that are larger than 1, counting multiplicities. 
Finally, let $(x, \xi) \in N$, where $\xi$ is a unit vector. For $t>0$, let $p=F(x, t \xi)$. Then $p$ is a focal point of $(M, x)$ if and only if $1 / t$ is an eigenvalue of $A_{\xi}$. Suppose $1 / t$ is not an eigenvalue of $A_{\xi}$. Then the function $L_{p}$ has $x$ as a nondegenerate critical point and the index at $x$ is equal to the number of positive eigenvalues (counting multiplicities) that are greater than $1 / t$.

We now prove a lemma which is crucial in the proof of our results.

LEMma. Let $p \in R^{m}$ and assume that the function $L_{p}$ has a nondegenerate critical point $x \in M^{n}$ of index $k$. Then there exists a point $q \in R^{m}$ such that $L_{q}$ is a Morse function which has a critical point $z$ of index k. ( $q$ and $z$ may be chosen as close to $p$ and $x$, respectively, as we want.)

Proof. Let $p=F(x, \xi)$, where $\xi$ is a normal vector at $f(x)$. By assumption, $p$ is not a focal point of $(M, x)$, that is, the Jacobian $F_{*}$ is nondegenerate at $(x, \xi)$. Thus there exists a neighborhood $U$ of $(x, \xi)$ in the normal bundle $N$ such that $F$ gives a diffeomorphism of $U$ onto a neighborhood $V=F(U)$ of $p$ in $R^{m}$. (Of course, $U$ and $V$ may be chosen as small as we like.) Now $V$ has a point $q$ such that $L_{q}$ is a Morse function (i.e., $q$ is not a focal point of $M$ ), because the set of focal points of $M$ has measure 0 . We have $q=F(z, \zeta)$ for some $(z, \zeta) \in U$. We show that the index of $L_{q}$ at $z$ is equal to $k$.

Consider a differentiable family of symmetric endomorphisms $I-A_{\eta}$ on $T_{y}\left(M^{n}\right)$, where $(y, \eta)$ runs over $U$. If we denote the eigenvalues by

$$
\lambda_{1}(y, \eta) \geqq \lambda_{2}(y, \eta) \geqq \cdots \geqq \lambda_{n}(y, \eta),
$$

then it can be shown that each $\lambda_{i}$ is a continuous function on $U$. Since $F_{*}$ is nondegenerate at each point of $U$, none of these functions takes value 1 on $U$. The index of $L_{p}$ at $x$ being $k$ by assumption, we have that $\lambda_{1}, \cdots, \lambda_{k}$ are greater than 1 at $(x, \xi)$ and $\lambda_{k+1}, \cdots, \lambda_{n}$ are less than 1 at $(x, \xi)$. It follows that the same arrangement holds at $(z, \zeta)$. This means that the index of $L_{q}$ at $z$ is equal to $k$. We have thus proved the lemma.

\section{Proof of Theorem B.}

Under the assumptions of Theorem B, we shall show the following fact. If $x \in M^{n}$ and if $\xi$ is a unit vector normal to $f\left(M^{n}\right)$ at $f(x)$, then 
$A_{\xi}=c I$ for some constant $c$, that is, $A_{\xi}$ has only one eigenvalue (of multiplicity $n$ ). Suppose $A_{\xi}$ has a non-zero eigenvalue, say, $a$. We may assume that $a>0$, because if $a<0$, then $A_{-\xi}$ has $-a>0$ as eigenvalue; if we can show that $A_{-\xi}=(-a) I$, then we know that $A_{\xi}=-A_{-\xi}=a I$.

Assuming thus that $a$ is the largest positive eigenvalue of $A_{\xi}$, take $t>0$ such that $1 / a<t<1 / b$, where $b$ is the next largest positive eigenvalue if any (if $a$ is the only positive eigenvalue, just consider $1 / a<t)$. Then $p=F(x, t \xi)$ is not a focal point of $(M, x)$ and the function $L_{p}$ has $x$ as a nondegenerate critical point. The index at $x$ is equal to the multiplicity, say, $k$, of the eigenvalue $a$. If $L_{p}$ is a Morse function, the assumption in Theorem $\mathrm{B}$ implies $k=n$, since $k$ cannot be 0 . Now $L_{p}$ may not be a Morse function (it can have a degenerate critical point elsewhere). By the lemma in Section 1, however, we know that there must exist a Morse function of the form $L_{q}, q \in R^{m}$, which has a critical point $z$ of index $k$. Thus we may conclude that $k=n$. This means that $a$ is an eigenvalue of $A_{\xi}$ with multiplicity $n$ so that $A_{\xi}=a I$.

What we have just shown implies that $M^{n}$ is umbilical, that is, if $\eta$ denotes the mean curvature vector field, then for any normal vector $\xi$ at $x$ we have

$$
A_{\xi}=\langle\xi, \eta\rangle I
$$

Equivalently, every $X \in T_{x}\left(M^{n}\right)$ is a principal vector in the sense that there exists a 1-form $\omega$ on the normal space $N_{x}$ such that

$$
A_{\xi}(X)=\omega(\xi) X \text { for all } \xi \in N_{x} \text { and } X \in T_{x}(M)
$$

It is known (see [1, p. 231]) that a complete Riemannian manifold isometrically and umbilically immersed in $R^{m}$ is actually imbedded as a Euclidean $n$-subspace or a Euclidean $n$-sphere. This completes the proof of Theorem B.

It is quite easy to derive Theorem A from Theorem B. If a Morse function $L_{p}$ has exactly two critical points, then one is where $L_{p}$ has a maximum (hence of index $n$ ) and the other is where $L_{p}$ has a minimum (hence of index 0 ). Thus every Morse function $L_{p}$ has index $n$ or 0 at a critical point.

Suppose $S^{n}$ is a Euclidean $n$-sphere in $R^{m}$ and assume we have taken a rectangular coordinate system $x_{1}, \cdots, x_{m}$ in $R^{m}$ so that 


$$
S^{n}=\left\{\left(x_{1}, \cdots, x_{n+1}, 0, \cdots, 0\right) ; \sum_{k=1}^{n+1} x_{k}^{2}=r^{2}\right\}
$$

Then we can see that the set of focal points of $S^{n}$ is the Euclidean $(m-(n+1))$-subspace defined by $x_{1}=\cdots=x_{n+1}=0$. If $p$ is not a focal point, the Morse function $L_{p}$ has exactly two critical points, one of index $n$ and the other of index 0 .

What we have just said is sufficient to derive Theorem $\mathrm{C}$ from Theorem B.

\section{Remarks.}

Our main results may be formulated without explicitly involving the notion of Morse functions and, indeed, under a weaker assumption. Let $D$ be a dense subset of $R^{m}$. In Theorems $\mathrm{A}, \mathrm{B}$ and $\mathrm{C}$, we may replace "every Morse function on $M^{n}$ of the form $L_{p}, p \in R^{m}$ " by "every function on $M^{n}$ of the form $L_{p}, p \in D$ ".

The proof of Theorem B under this weaker assumption remains almost the same as before except for a corresponding change in the lemma, namely, the conclusion of the lemma should be modified as follows: "Then there exists a point $q \in D$ such that $L_{q}$ has a critical point $z$ of index $k$."

Finally, we note that if $M^{2}$ immersed in $R^{m}$ is topologically a 2sphere, then our original assumption in Theorem $\mathrm{A}$ is equivalent to the spherical two-piece property studied by T. F. Banchoff: The spherical two-piece property and tight surfaces in spheres, J. Differential Geometry 4(1970), 193-205 (see, in particular, Theorem 3).

\section{REFERENCES}

[1] É. Cartan, Leçons sur la géométrie des espaces de Riemann, deuxième édition, Gauthier-Villars, Paris, 1946.

[2] J. Milnor, Morse Theory, Ann. of Math. Studies, No. 51, Princeton University Press, 1963.

Brown University 